\title{
The Abuse of Dermal Fillers: A Clinico-Epidemiological Study of 35 Cases
}

\author{
Nabeel K. Al Hamzawi ${ }^{*}$, Salih M. Al Baaj ${ }^{2}$ \\ ${ }^{1}$ Department of Dermatology, Diwaniyah Teaching Hospital, Diwaniyah, Iraq \\ ${ }^{2}$ Department of Plastic Surgery, Diwaniyah Teaching Hospital, Diwaniyah, Iraq \\ Email: *alhamzawi_n@yahoo.com
}

How to cite this paper: Al Hamzawi, N.K. and Al Baaj, S.M. (2019) The Abuse of Dermal Fillers: A Clinico-Epidemiological Study of 35 Cases. Journal of Cosmetics, Dermatological Sciences and Applications, 9, 94-106.

https://doi.org/10.4236/jcdsa.2019.92008

Received: January 24, 2019

Accepted: April 7, 2019

Published: April 10, 2019

Copyright $\odot 2019$ by author(s) and Scientific Research Publishing Inc. This work is licensed under the Creative Commons Attribution International License (CC BY 4.0).

http://creativecommons.org/licenses/by/4.0/

\begin{abstract}
Background: Nowadays, the use of dermal fillers for correcting signs of aging, such as wrinkles, depressions, and folds, has become increasingly popular. However, the abuse of these products can lead to disfigurements and unpredictable consequences among users. Objective: To evaluate the clinical abnormalities related to dermal fillers abuse in Iraqi people, and identify the various factors contributing to them. Methods: A total of 35 patients with different facial abnormalities due to the abuse of dermal fillers, attending our clinic in Diwaniyah, Iraq, from September 2016 to December 2017, were enrolled. A questionnaire-based analysis was used to collect clinical and sociodemographic data from patients to determine the etiological profile behind this problem. Several disfigurements and facial defects were diagnosed on clinical base. We have treated most of the situations that require intervention and treatment. Results: A total of 35 patients aged between 25 and 54 years with a sex ratio of 0.093 were enrolled in this study. Their mean age was 42 years, and the age group between 35 and 45 years was forming $85.7 \%$ of the cases. The clinical abnormalities recorded in this study include: buffy face 8 (22.8\%), facial asymmetry $3(8.5 \%)$, Mdoka fish lips like $6(17 \%)$, periorbital redness $1(2.8 \%)$, filler fatigue $6(17 \%)$, bags under eyes $4(11.4 \%)$, sizeable bulbous nose 4 (11.4\%), topographical changes 2 (5.7\%), and stone-like cheeks $1(2.8 \%)$. The leading causes for the abuse of fillers were as follows: 12 (34.28\%) candidates were not compatible with the procedure, $9(25.7 \%)$ were non-compliance with the indications of the Food and Drug Organization (FDA), 9 (25.7\%) fillers were injected in the wrong place, 18 (51.4\%) injections were of a larger quantity than required, and 5 (14.2\%) had frequent and long-term injection. Conclusion: The use of dermal fillers for a wrong purpose can result in disfigurements rather than beautification. The commonest disfigurements are the puffy face, enlarged lips, and bags under eyes. The
\end{abstract}


clinical uses of dermal fillers in Iraq need to be monitored by the health authorities, with the assurance that these products should be sold only by a doctor's prescription to reduce their abuse.

\section{Keywords}

Dermal Filler, Abuse, Clinical Abnormalities, Disfigurements

\section{Introduction}

Dermal fillers are synthetic or naturally derived products that are injected into the skin to enhance correction of wrinkles, depressions, and folds. However, with the manufacturing of new types, the indications of dermal fillers are increasingly rising to include tissue contouring and replacement of any lost volume in the skin or subcutaneous fat. Further indications of fillers include tear trough correction, reshaping of the nose and chin, cheek augmentation, lip enhancement, hand rejuvenation, and the correction of facial asymmetry. Recently, fillers have come to play an essential role in the nonsurgical management of aging skin [1] [2]. According to Statistical data from American Society for Aesthetic Plastic Surgery (ASAPS), more than 2 million dermal filler procedures were performed in 2015 [3]. Due to the increased demand for dermal fillers, the consumption of them annually increases, so does their number in the market; more than 150 products are currently available worldwide for 50 companies [4]. As in any medical procedure, there are expected risks which can occur with the use of dermal fillers. Therefore, it is crucial for the injector to understand their limits and possible risks. The causes behind these risks are multifactorial and may include the followings: 1) Non-certified individual or non-trained practitioner; 2) The use of unapproved products; 3 ) Improper injection techniques can be associated with a high incidence of adverse events [5]. Thus, the injector should be familiar with the facial anatomy, the physical properties of fillers, and various types of injections techniques to increase the safety potential of the procedure. There are many studies about the traditional complications of filler, but to our knowledge, this is the first work dealing with the abuse topic. We have done this work to draw the attention of cosmetic professionals to the unfortunate consequences of this procedure. This study aimed to evaluate the clinical abnormalities related to dermal fillers abuse in Iraqi people and identify the various factors contributing to them.

\section{Patients and Methods}

A total of 35 patients participated in our dermatology and laser clinic in the province of Diwaniyah, Iraq, during the period from September 2016 to December 2017, were enrolled for this study after taking their approval. Inclusion criteria for patients were the presence of facial disfigurements and clinical ab- 
normalities one month after filler injection.

Patients with common complications, patients who received combination therapy such as filler and Botox, and those who were taken treatment after the procedure, were excluded from the study. A questionnaire-based analysis consists of two sections, the first to collect sociodemographic details and the second to obtain clinical information, has been used. Each patient interviewed about the age, the place of residence (rural or urban), the level of education, and the presence of any health problems. Clinical data included the reason for the use of filler, the duration and frequency of injection, the site of injection, the amount of filler injected, the trade name of the product used, and who did the procedure and where it was performed in a clinic or beauty center, have been recorded. A detailed physical examination was performed including skin and systemic analysis. Routine laboratory investigations were carried out to exclude health problems. The diagnosis of various abnormalities and facial defects was made on clinical base.

Regarding the treatment, patients with overcorrection were given hyaluronidase injection to dissolve the excess fillers. Patients with allergic reaction prescribed systemic corticosteroids and antihistamines in addition to hyaluronidase. The follow-up continued for these patients until satisfactory results were obtained.

\section{Results}

\subsection{Study Limitations}

We were confronted with some difficulties during this study. Several patients do not have sufficient information about the name or quantity of the product used. Others agreed to conduct the study but refused to take photos. We lost contact with five patients after treating them. This work was done on a sample in one clinic for 15 months. The real number will be much higher if extensive studies are to be performed in the entire country, because of the great craze for using fillers in these age groups.

\subsection{Epidemiological Aspect}

A total of 35 patients were evaluated in this study, female patient $32(91.42 \%)$ were more predominant than male patient $3(8.58 \%)$, with a sex ratio of 0.093 . Their average age was 42 years, ranged from 25 to 54 years. The age groups from 35 to 45 years were forming $85.7 \%$ of cases (Table 1 ).

The leading causes for the abuse of fillers in all patients listed as follows: 12 (34.28\%) candidates are not compatible with the procedure, 9 (25.7\%) non-compliance with the indications of the Food and Drug Organization (FDA), 9 (25.7\%) filler injected in a wrong place, 18 (51.4\%) injection of a large quantity than required,

Table 1. Distribution of cases was in term of sex and age group.

\begin{tabular}{cccccc}
\hline Patient & $21-30$ & $31-40$ & $41-50$ & $>50$ & Total \\
\hline Male & 0 & 1 & 2 & 0 & 3 \\
Female & 2 & 17 & 10 & 3 & 32 \\
\hline
\end{tabular}


5 (14.2) frequent and long-term injection, 15 (42.8\%) non-trained hands, 17 (48.5\%) lack of scientific knowledge, 13 (37.1\%) the use of unapproved products. In each patient, we found several causes for the abuse of filler; hence, the total number of contributing factors exceeded the number of patients (Table 2).

\subsection{Clinical Aspect}

The clinical abnormalities recorded in this study include (Table 3):

1) Buffy face or helium balloon: 8 (22.8\%) of the cases presented with puffiness of their faces, one of these patients, a 35-year-old woman formerly has a moon face as a result of taking dexamethasone tablets, and was subjected to filler injection in a beauty center. One month later she presented with puffiness and redness of the whole face (Figure 1(a)). The second case, a 52-year-old woman, came with huge cheeks six weeks after the injection (Figure 1(b)). The third one, a 43-year-old lady presented with massive facial edema following injection of a large amount of filler (Figure $1(\mathrm{c})$ ).

2) Facial asymmetry; 3 (8.5\%) of the cases were suffering from a lack of similarity of the sides of the face, two of them demonstrated in (Figure 2(a) and Figure 2(b)).

Table 2. The proportion of etiological factors in the cases.

\begin{tabular}{ccc}
\hline Etiological factor & Patients No. & $\%$ \\
\hline $\begin{array}{c}\text { Candidates are not suitable for the procedure } \\
\text { Non-compliance with the (FDA) indication }\end{array}$ & 12 & $34.28 \%$ \\
Injection of a large quantity than required & 9 & $25.7 \%$ \\
Frequent and long-term injection & 18 & $51.4 \%$ \\
Non-trained hands & 5 & $14.2 \%$ \\
Lack of scientific knowledge & 15 & $42.8 \%$ \\
The use of (FDA) unapproved products & 17 & $48.5 \%$ \\
\hline
\end{tabular}

Table 3. The proportion of various clinical abnormalities in the sample.

\begin{tabular}{ccc}
\hline Clinical abnormality & Number & Proportion \\
\hline Buffy face & 8 & $22.8 \%$ \\
Facial asymmetry & 3 & $8.5 \%$ \\
Mdoka fish lips like & 6 & $17 \%$ \\
Periorbital redness & 1 & $2.8 \%$ \\
Filler fatigue & 6 & $17 \%$ \\
Bags under eyes & 4 & $11.4 \%$ \\
Sizeable bulbous nose & 4 & $11.4 \%$ \\
Topographical changes & 2 & $5.7 \%$ \\
Stone-like cheeks & 1 & $2.8 \%$ \\
\hline
\end{tabular}




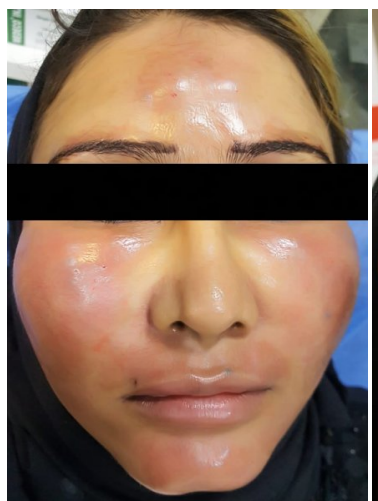

(a)

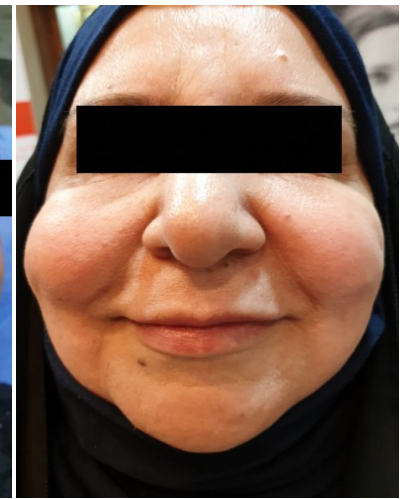

(b)

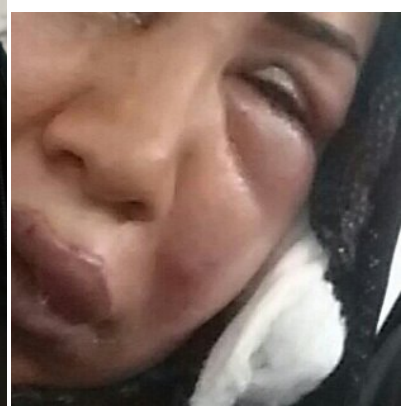

(c)

Figure 1. (a) A 35-year-old woman formerly has a moon face as a result of taking dexamethasone tablets and was subjected to filler injection in a beauty center; (b) A 52-year-old woman, came with huge cheeks six weeks after the injection; (c) Facial edema due to due to injection of filler in large quantity than required.

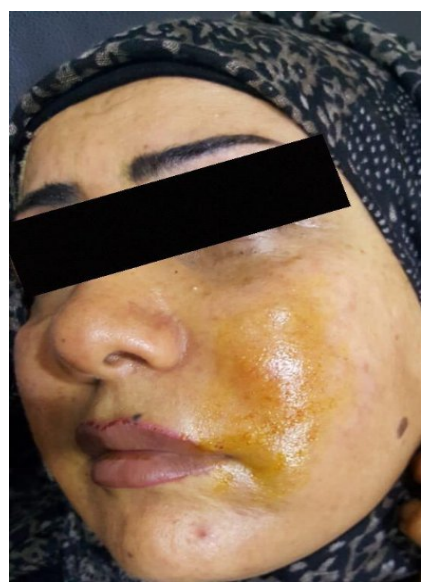

(a)

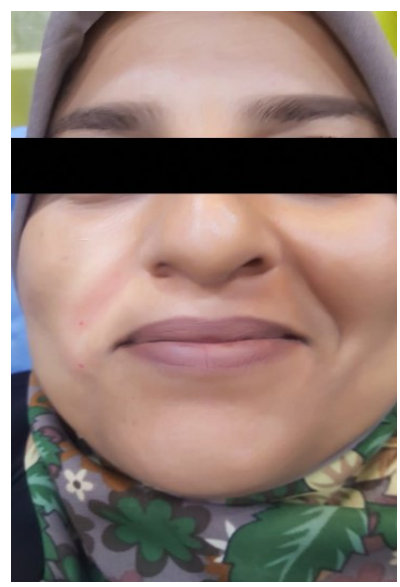

(b)

Figure 2. (a) A 41-year-old woman with facial asymmetry due to injection of filler in a wrong place; (b) A 37-year-old woman with facial asymmetry.

3) Mdoka fish lips like; 6 (17\%) of the patients have large lips that exceeded the limits of aesthetic measurements, i.e. (Golden ratio of the upper to lower lip $1: 1.618)$. One lady came with the upper lip is larger than the lower, and another two with oversized lips (Figures 3(a)-(d)). All of them looks similar to the lips of Mdoka fish that belongs to Placidochromis phenochilus (is a rare fish of cichlid species endemic to the northern parts of Lake Malawi, characterized by distinctive lips used for fighting and picking up objects) (Figure 3(e)).

4) Periorbital redness; 1 (2.8\%): a 46-year-old woman presented with bilateral redness around her eyes, due to an allergic reaction to hyaluronic acid material (Figure 4).

5) Filler fatigue: $6(17 \%)$ of cases came with tired faces. One of them, a 47-year-old woman, was subjected to four previous sessions of fillings within three years (Figure 5 ).

6) Bags under eyes: 4 (11.4\%) had bags under eyes. One of them 36-year-old 


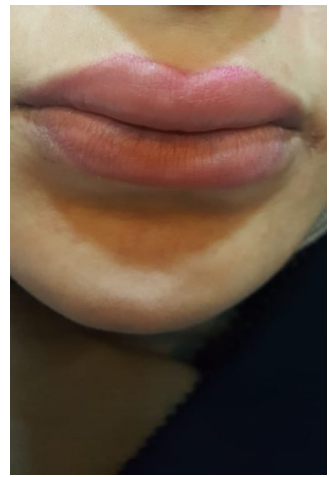

(a)

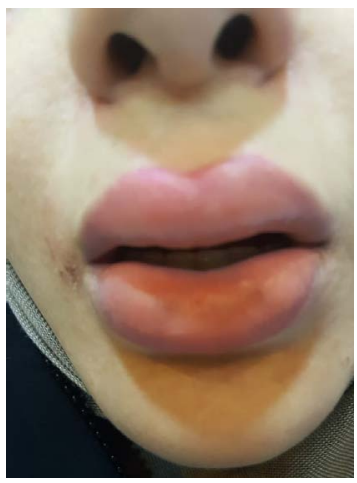

(b)

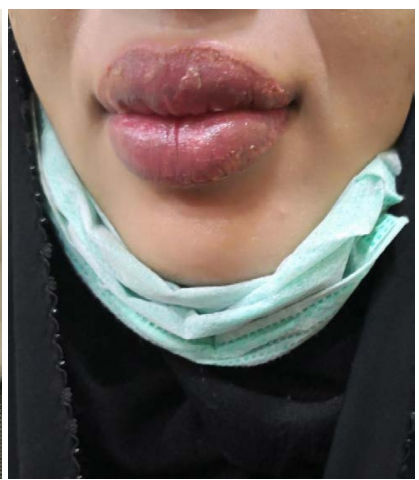

(c)

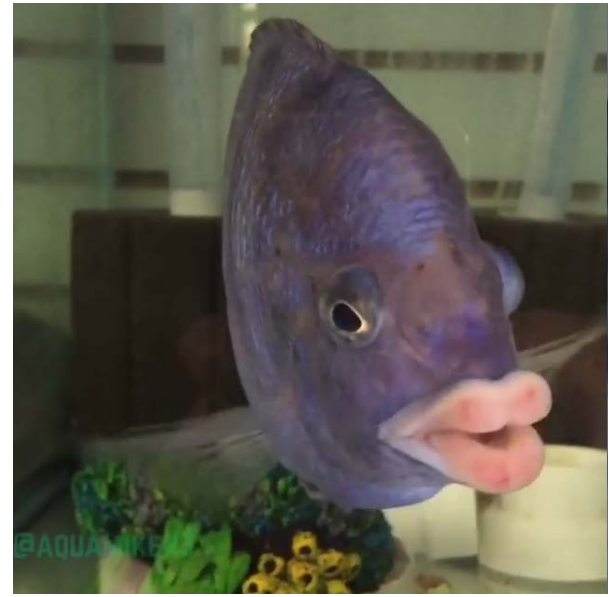

(e)

Figure 3. (a) and (b) A 33-year-old woman with swollen lips, one month after injection; (c) A 29-year-old woman with puffy lips and overdrawing; (d) Although she had plumbed lips, she asked for another dose for filler; (e) Mdoka fish belongs to Placidochromis phenochilus is a species of cichlid endemic to the northern parts of Lake Malawi. Reproduced from http://wsbuzz.com/world-news/meet-fish-perfect-pout-thanks-lips-look-almost-human/.

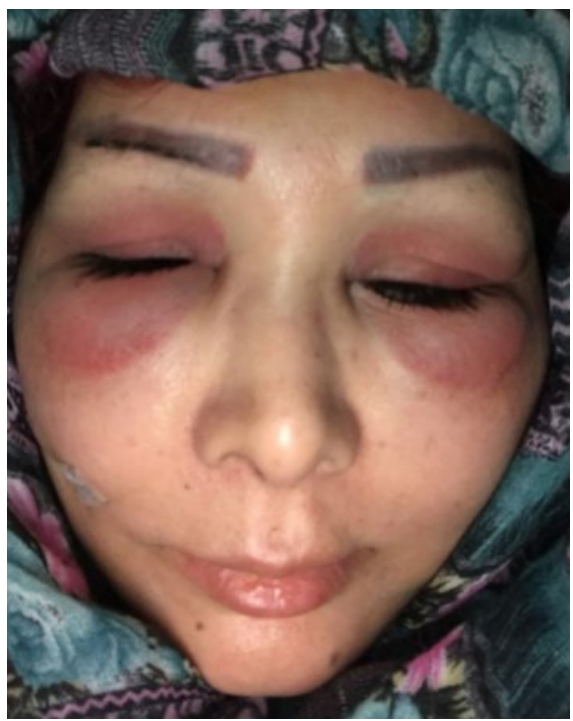

Figure 4. A 46-year-old woman presented with bilateral redness and swelling around her eyes, after injection of large quantity than required. 
women presented with bilateral bags under eyes four weeks after injections (Figure 6(a)). The bags disappeared by injecting hyaluronidase $30 \mathrm{IU}$ into each one. $150 \mathrm{IU}$ of hyaluronidase were dissolved in $1 \mathrm{ml}$ saline solution $(0.9 / \mathrm{Na} \mathrm{Cl})$, $0.2 \mathrm{ml}$ of the dissolved drug injected into each side by local infiltration with $32 \mathrm{G}$ needle using microdroplet technique (Figure 6(b)).

7) Sizeable bulbous nose: 4 (11.4\%) of cases came with a large nose, among them a girl of 25-year-old who was suffering from a nasal birth defect, that was injected with a large amount of filler (Figure 7).

8) Topographical changes: $2(5.7 \%)$ one of them, a 52 -year-old woman was suffering from changes in her facial topography due to the migration of excess

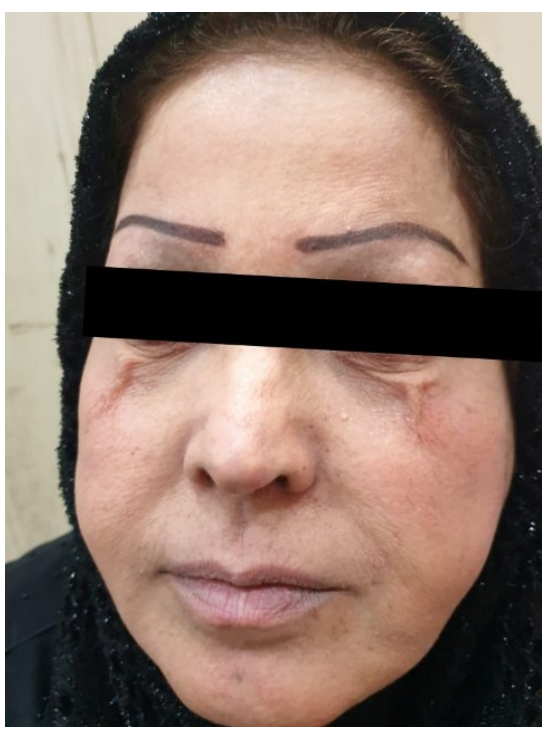

Figure 5. A 51-year-old woman with apparent fatigue after she had exposed to four sessions of filler injection within three years.

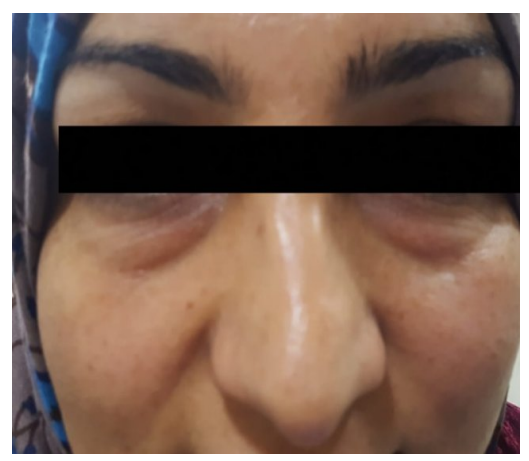

(a)

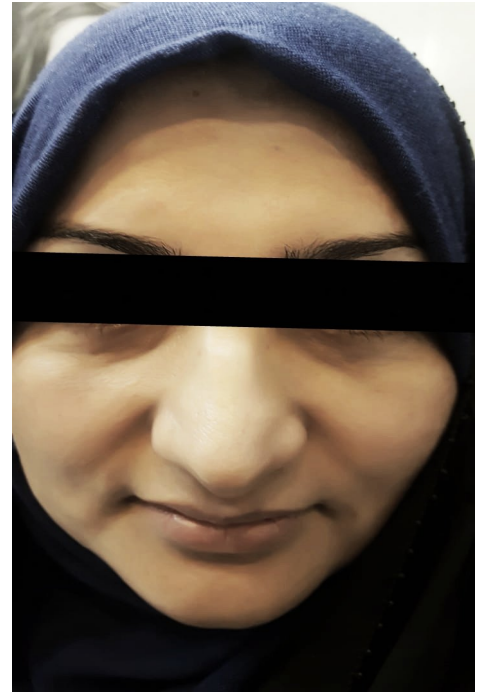

(b)

Figure 6. (a) Bags under eyes in 36-year-old woman due to injection of a large amount of filler; (b) Disappearance of bags under eyes following hyaluronidase injection. 
filler from the cheeks to the lower face and jawlines, in a way that made the width of the bottom of the face more than the top (Figure 8).

9) Stone-like cheeks: $1(2.8 \%)$ a 43 -year-old woman came with oversized, stone-like cheeks after receiving repeated injections of fillers during two years, the patient presented 4 months after the last session (Figure 9).

\subsection{Therapeutic Aspect}

Hyaluronidase injection was administered to most of the patients to dissolve the excess fillers and restore their natural appearance. The dose ranged between 30 to $75 \mathrm{IU}$ according to the site and size of the treated abnormality. Patient with

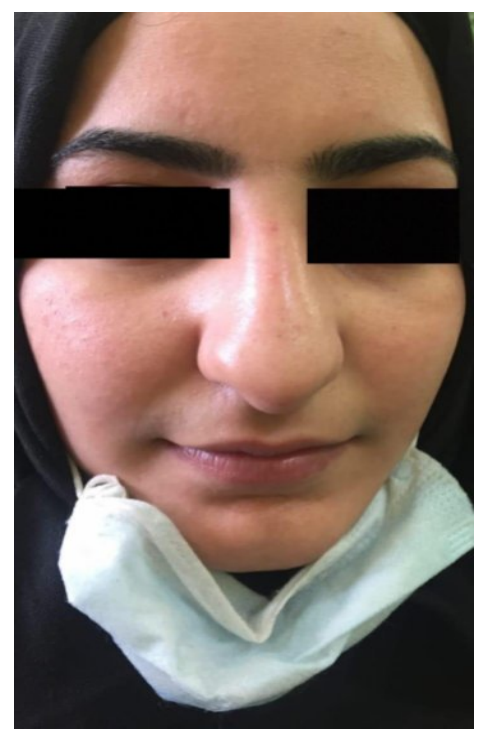

Figure 7. A 25-year-old female with large nose due to overcorrection of a birth defect with filler.
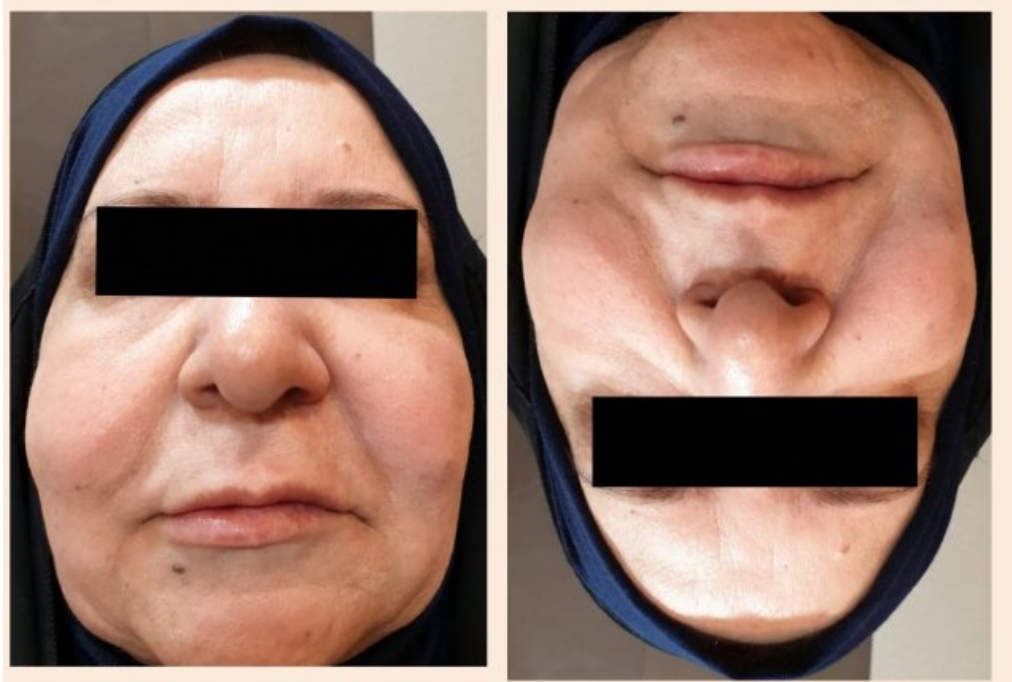

Figure 8. A 52-year-old woman with marked changes in her facial topography due to the migration of excess filler from the cheeks to the lower face and jaw lines, making the image of the face as if inverted. 
facial edema and puffy face prescribed systemic corticosteroids for 5 days, then injected with hyaluronidase. Patient with periorbital redness treated with systemic steroid after exclusion of medical contraindications. Patient with stone-like cheeks treated with intralesional triamcinolone injection to reduce the hard granulomatous tissue. For each patient follow-up was continued for one month after treatment until a satisfactory result was achieved.

By asking patients about the places where the procedure performed, the people who injected them, the brand name of the product used, and how many syringes were used. We found that $15(42.8 \%)$ of cases were conducted in beauty centers, 11 (31.4\%) by cosmetic physicians, 7 (20\%) by dental clinics, and 2 (5.7\%) by barber salons (Table 4). Some patients mentioned a name of products that were not approved by the FDA, but the majority of them did not know the name of the used product.

\section{Discussion}

The burden of dermal fillers abuse is not known because there is no recording and documentation of cases. This study was conducted over 15 months, to record the etiological and clinical aspects associated with the abuse of dermal fillers in Iraqi people, as these procedures are considered new in our country. The

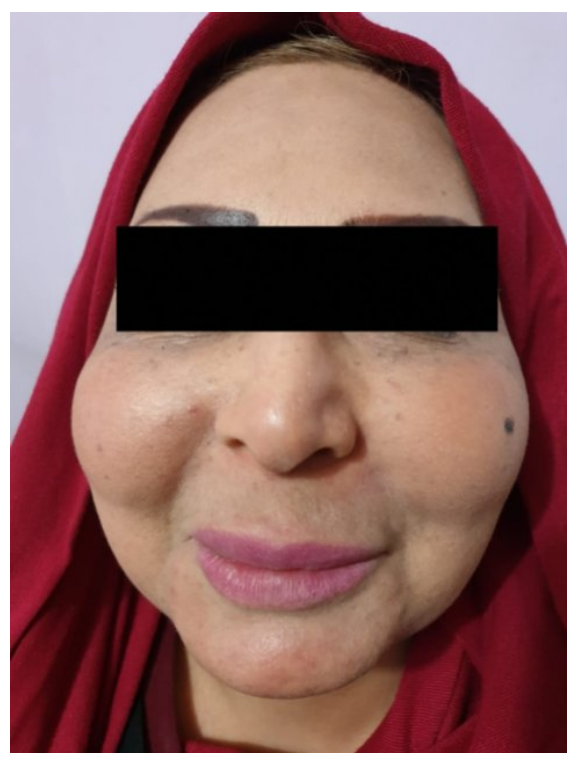

Figure 9. A 43-year-old woman came with oversized, stone-like texture cheeks after injecting them with repeated fillers throughout two years.

Table 4. The number of patients per the place where the procedure was performed.

\begin{tabular}{ccc}
\hline Place of procedure & Patients No. & $\%$ \\
\hline Beauty center & 15 & $42.8 \%$ \\
Cosmetic physician & 11 & $31.4 \%$ \\
Dental clinic & 7 & $20 \%$ \\
Barber salon & $\mathbf{2}$ & $\mathbf{5 . 7 \%}$ \\
\hline
\end{tabular}


age of patient included in this study ranged between 25 to 54 years. Among the patients studied, we found that the highest prevalence (85.7\%) were people aged between their late $30 \mathrm{~s}$ and early $40 \mathrm{~s}$. All patients in this study were residents of the city, and none of them were from the rural areas, so this work considered as an urban population-based study. The increasing commercial advertisements of beauty centers and cosmetic physicians on social media have an impact on the culture of these people. As a result, some of them ask for filler even though there is no need for them to use it. The doctor should assess the patient's demands and do the procedure within the real indications, and not follow the patient's wishes. Some patients want to correct some errors and restore their previous looks (especially in middle age) while others want to change their looks (especially young adults and teens). The right opinion is to achieve the most natural look for years to come, not too filled or overly treated. Most of the present cases in this study underwent filling procedures in beauty centers, dental clinics, and even barber salons.

Dermal fillers may be the best choice for many people; however, they may become like a bane to others. What is essential in this procedure is to choose the right filler for the right place in a fit candidate [6]. In Iraq, about half of the filler procedures are being conducted in substandard clinics and beauty salons in the absence of health control, and this has led to an increase in the number of abuse cases. One of the leading causes for the abuse is the conduction of candidate who is not compatible with the procedure such as people over 50 years with excessive sagging or severe wrinkling of the skin. As such people need a large amount of filler to ensure visible correction; consequently, the results will be unsatisfactory. Dermal fillers are used to treat mild and medium wrinkles, which result from the decay of the collagen layer with age, and this is often present in the late 30s and early 40s. It also used to correct scars and defect resulting from lipoatrophy [7] [8] [9]. Thus, it is considered abuse, when injected for people with severe sagging and wrinkling of the skin. Fillers cannot correct every defect or unsatisfactory appearance; it is important to select patients who have FDA-approved indications to avoid any problems for them. For example patient with a nasal birth defect is an indication for rhinoplasty, and cannot be corrected by filler as it may give rise to more ugly appearance, as what happened to the patient in (Figure 7).

Moreover, the injection at a site that is not indicated by the FDA such as breasts, buttocks, and foot may carry a risk of complications such as infection, granuloma, and pulmonary embolization especially when the large amount was injected [10]. The injection of the materials in large quantities than needed is counterproductive because, with time, fillers begin to dissolve, leading to sagging of the skin, which increase the need for filler again. The frequent injections of temporary fillers, especially in people over 45 years of age, lead to so-called filler fatigue, once the material dissolved (Figure 5). Furthermore, repeating facial tiredness and fatigability requires further visits that will cost the patient. The frequent and long-term use of fillers can lead to the hardening of the subcutaneous 
tissues, as fillers, whenever injected will trigger inflammation in the area, followed by tissue fibrosis [11] [12]. The frequency exceeds one injection per year, and the phrase "long-term" implies the repetition of the procedure each year for up to four to five years. Factors like the amount, the types, and the physical properties of fillers may enhance the development of foreign body granulomas which can be divided into three classes based on clinical features: cystic granulomas, edematous granulomas, and sclerosing granulomas.

Moreover, mixed type foreign body granulomas sometimes occur [13]. This explains to us that the stone-like cheeks in (Figure 9) happened due to tissue fibrosis and granuloma formation. It is a scientific fact that the lost tissue should replace by the tissue of similar texture, i.e., skin collagen by filler, subcutaneous fat by fat, muscle by muscle and so on. People who are suffering from the loss of subcutaneous fat, the best option for them is the fat transfer instead of filler because it can maintain more support, for a longer duration. While those with excessive sagging due to aging and the effect of gravity, they need lifting to correct their errors. Giving a high dose of fillers in such conditions can lead to disfigurement rather than beautification.

The use of dermal fillers to draw a new look is a matter of art that requires artistic eyes and well-trained hands. It is wrong to inject fillers based on the desire of the patient to appear with certain cheeks or lips. Because the dimensions of these parts may be acceptable in the concept of gold ratio, and using a filler will change the areas and make them reverse to the accepted rate. The oversized lips are seen in (Figure 3), an example of injection that was done in a beauty center according to the patient's desire. It resulted in the formation of lips that simulated Mdoka White Lips (a rare fish of the species Placidochromis phenochilus). The causes behind this abnormality are the injection of large amount than needed and placement of the material in the pink part of the lip rather than the vermilion border.

For safer results, the physician should choose the FDA-Approval fillers and others that allowed by the approval system of the ministry of health. The injector must know the different techniques used for injection, depot, push-ahead, threading, fanning, and cross-hatching. The secret of success in this procedure is to deposit the correct amount at the right depth. Our recorded cases of abuse, especially those with puffiness of the cheeks, bags under the eyes, and swollen lips were treated with hyaluronidase injection. Hyaluronidase is effective to treat the overcorrection, but it may trigger allergies after its use, thereby, the therapist must do allergic skin testing before giving this enzyme to avoid the risk of allergy and/or angioedema [14] [15].

In Iraq, the abuse of dermal fillers comes as a result of the absence of health control on the unauthorized beauty centers and local companies that import products of low quality, which pose a threat to public health. We suggest that the best solution to limit this problem is to consider dermal filler as a medicine, and not a cosmetic substance so that it is taken out of the hands of local companies, 
and sold in pharmacies, after being subject to quality control as other medications. In this case, only globally licensed types will be used, and the drug will be prescribed according to the real need. The patient can retain the prescription with all information about the nature and quantity of the product. Based on what is mentioned, we believe that the previous step will reduce abuse of the fillers by the beauty centers and low-caliber physicians.

\section{Conclusion}

The use of dermal fillers for a wrong purpose can result in disfigurements rather than beautification. We have concluded that the most common causes of abuse are the selection of candidates who are not compatible with the procedure, the untrained hands and the use of low-quality types of fillers to tempt victims at low prices. The use of these products in Iraq needs to be monitored by the health authorities, with the assurance that these materials should be sold only by a doctor's prescription to reduce their abuse.

\section{Conflicts of Interest}

None declared.

\section{References}

[1] Vedamurthy, M. (2008) Standard Guidelines for the Use of Dermal Fillers. Indian Journal of Dermatology, Venereology \& Leprology, 74, 23-27.

https://www.ncbi.nlm.nih.gov/pubmed/18688100

[2] Goldberg, D.J. (2006) Legal Ramifications of Off-Label Filler Use. Dermatologic Therapy, 19, 189-193. https://doi.org/10.1111/j.1529-8019.2006.00073.x

[3] The American Society for Aesthetic Plastic Surgery (2017) Cosmetic Surgery National Data Bank Statistics. https://www.surgery.org/media/statistics

[4] Funt, D. and Pavicic, T. (2013) Dermal Fillers in Aesthetics: An Overview of Adverse Events and Treatment Approaches. Clinical, Cosmetic and Investigational Dermatology, 6, 295-316. https://doi.org/10.2147/CCID.S50546

[5] Glogau, R.G. and Kane, M.A. (2008) Effect of Injection Techniques on the Rate of Local Adverse Events in Patients Implanted with Nonanimal Hyaluronic Acid Gel Dermal Fillers. Dermatologic Surgery, 34, S105-S109. https://doi.org/10.1111/j.1524-4725.2008.34251.x

[6] Vedamurthy, M. and Vedamurthy, A. (2008) Dermal Fillers: Tips to Achieve Successful Outcomes. Journal of Cutaneous and Aesthetic Surgery, 1, 64-67. https://doi.org/10.4103/0974-2077.44161

[7] Gerald O'Danie, T. (2011) Multimedia Management of Atrophic Acne Scarring in the Aging Face. Aesthetic Plastic Surgery, 35, 1143-1150. https://doi.org/10.1007/s00266-011-9715-y

[8] Wollina, U. and Goldman, A. (2015) Fillers for the Improvement in Acne Scars. Clinical, Cosmetic and Investigational Dermatology, 8, 493-499. https://doi.org/10.2147/CCID.S86478

[9] Requena, L., Requena, C., Christensen, L., Zimmermann, U.S., Kutzner, H., Cerroni, L. (2011) Adverse Reactions to Injectable Soft Tissue Fillers. JAAD, 64, 1-34. https://doi.org/10.1016/j.jaad.2010.02.064 
[10] Styperek, A., Bayers, S., Beer, M. and Beer, K. (2013) Nonmedical-Grade Injections of Permanent Fillers: Medical and Medicolegal Considerations. Journal of Clinical and Aesthetic Dermatology, 6, 22-29.

[11] Pravin, M., Romain, K., Sana, B., Laurence Toutous, T., Alexandra, P. and Minerva, B. (2017) Injectable Facial Fillers: Imaging Features, Complications, and Diagnostic Pitfalls at MRI and PET CT. Insight Imaging, 8, 557-572.

https://doi.org/10.1007/s13244-017-0575-0

[12] Haneke, E. (2015) Managing Complications of Fillers: Rare and Not-So-Rare. Journal of Cutaneous and Aesthetic Surgery, 8, 198-210. https://doi.org/10.4103/0974-2077.172191

[13] Lee, J.M. and Kim, Y.J. (2015) Foreign Body Granulomas after the Use of Dermal Fillers: Pathophysiology, Clinical Appearance, Histological features, and Treatment. Archives of Plastic Surgery, 42, 232-239. https://doi.org/10.5999/aps.2015.42.2.232

[14] Ranneva, E. (2017) The Use of Hyaluronidase to Treat the Excess of Cross-Linked Hyaluronic Acid Following Aesthetic Medicine Procedures: A Practical Point of View. Emerg Med (Los Angel), 7, 357. https://doi.org/10.4172/2165-7548.1000357

[15] Andre, P. and Flechet, M.L. (2008) Angioedema after Ovine Hyaluronidase Injection for Treating Hyaluronic Acid Overcorrection. Journal of Cosmetic Dermatology, 7, 136-138. https://doi.org/10.1111/j.1473-2165.2008.00377.x 